\title{
Improved Online Algorithms for 1-Space Bounded 2-Dimensional Bin Packing
}

\author{
Yong Zhang ${ }^{1,2 \star}$, Jingchi Chen ${ }^{2}$, Francis Y.L. Chin ${ }^{2 \star \star}$, Xin Han ${ }^{3 \star \star \star}$, \\ Hing-Fung Ting ${ }^{2 \dagger}$, and Yung H. Tsin ${ }^{4 \ddagger}$ \\ 1 College of Mathematics and Computer Science, Hebei University, China. \\ 2 Department of Computer Science, The University of Hong Kong, Hong Kong \\ \{yzhang, jchen, chin, hfting, \}@cs.hku.hk \\ 3 School of Software, Dalian University of Technology, China \\ hanxin.mail@gmail.com \\ ${ }^{4}$ School of Computer Science, University of Windsor, Canada \\ peter@uwindsor.ca
}

\begin{abstract}
In this paper, we study 1-space bounded 2-dimensional bin packing and square packing. A sequence of rectangular items (square items, respectively) arrive over time, which must be packed into square bins of size $1 \times 1.90^{\circ}$-rotation of an item is allowed. When an item arrives, we must pack it into an active bin immediately without any knowledge of the future items. The objective is to minimize the total number of bins used for packing all the items in the sequence. In the 1-space bounded variant, there is only one active bin for packing the current item. If the active bin does not have enough space to pack the item, it must be closed and a new active bin is opened.

Our contributions are as follows: For 1-space bounded 2-dimensional bin packing, we propose an online packing strategy with competitive ratio 5.155 , surpassing the previous 8.84-competitive bound. The lower bound of competitive ratio is also improved from 2.5 to 3 . Furthermore, we study 1-space bounded square packing, which is a special case of the bin packing problem. We give a 4.5-competitive packing algorithm, and prove that the lower bound of competitive ratio is at least $8 / 3$.
\end{abstract}

\section{Introduction}

The bin packing problem $[1-9,11-16,19,17,18,20-24]$ has been well studied for more than thirty years. In the general bin packing problem, a sequence of items are packed into bins without overlapping. The objective is to minimize the number of bins used for packing all the items in the sequence.

\footnotetext{
* Supported by Shanghai Key Laboratory of Intelligent Information Processing, China. Grant No. IIPL-2010-010

** Research supported by HK RGC grant HKU-7113/07E and the William M.W. Mong Engineering Research Fund

*** Partially supported by Start-up Funding (1600-852011) provided by DUT, China

$\dagger$ Research supported by HK RGC grant HKU-7171/08E

¥ Research supported by NSERC under grant NSERC 7811-2009
} 
Most previous studies do not impose a limit on the number of bins available for packing the items (called active bins). We call this model unbounded space. There is another model called bounded space, which is more realistic in many applications. In the bounded space model, the number of active bins is bounded by a constant, and each item can only be packed into one of the active bins. If none of the active bins has enough space to pack an item, one of the current active bins is closed and a new active bin is opened to pack that item.

In this paper, we consider 1-space bounded 2-dimensional bin packing and square packing, which are interesting variants of bin packing. In the 1-space bounded variant, the number of active bins is only one. If an item cannot be packed into the active bin, we have to close this bin and open a new one to pack the item. In the 1-space bounded 2-dimensional bin packing problem, each item is rectangular in shape and its width and height are no more than 1 . The items must be packed into square bins of size $1 \times 1.90^{\circ}$-rotation of any item is allowed, otherwise, the competitive ratio is unbounded [12]. 1-space bounded square packing is a special case of 1-space bounded 2-dimensional bin packing, where each item is a square with edge length no more than 1. Again, the objective is to minimize the number of square bins used.

For example, as shown in Fig. 1(a), there are four items to be packed into unit square bins, and the arrival order is $A, B, C$ and $D$. After the packing position of $A$ is fixed, we have two choices to pack $B$ : rotation and without rotation. If we pack $B$ without rotation in the same bin with $A$ as shown in Fig. 1(b), when item $C$ arrives, we have to open a new bin since the current active bin does not have enough space for packing $C$. In the optimal solution, these four items can be packed into one bin (Fig. 1(c)), since item $B, C$ and $D$ can be rotated and the free space in the bin can accommodate all of them in their order of arrival.

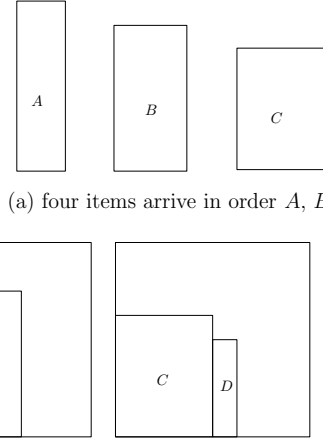

(b) non-optimal packing into two bins
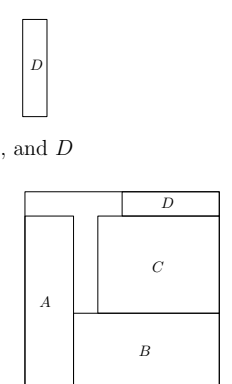

(c) optimal packing into one bin

Fig. 1. Example of optimal packing and non-optimal packing

We focus on the online version of 1-space bounded 2-dimensional bin packing and square packing, where the items arrive over time, and when packing the 
current item, we have no information of the future items and the position of the packed items in the bin cannot be changed. To measure the performance of online bin packing, the general method is to use the asymptotic competitive ratio. Consider an online algorithm $A$ and an optimal offline algorithm $O P T$. For any sequence $S$ of items, let $A(S)$ be the cost (number of square bins used) incurred by algorithm $A$ and $O P T(S)$ be the corresponding optimal cost incurred by algorithm $O P T$. The asymptotic competitive ratio for algorithm $A$ is:

$$
R_{A}^{\infty}=\lim _{k \rightarrow \infty} \sup _{S}\left\{\frac{A(S)}{O P T(S)} \mid O P T(S)=k\right\} .
$$

\section{Related works:}

Both the offline and online version of the bin packing problem have been well studied. For the offline version of two-dimensional bin packing. Chung et. al. [5] presented an approximation algorithm with an asymptotic performance ratio of 2.125. Caprara [4] improved the upper bound to 1.69103. Bansal et al. [2] devised a randomized algorithm with an asymptotic performance ratio of at most 1.525. As for the offline lower bound of the approximation ratio, Bansal et al. [1] showed that the two-dimensional bin packing problem does not admit any asymptotic polynomial time approximation scheme.

For online one-dimensional bin packing, Johnson et al. [16] showed that the First Fit algorithm (FF) has an asymptotic competitive ratio of 1.7. Yao [24] improved the algorithm to obtain a better upper bound of 5/3. Lee et al. [17] introduced the class of Harmonic algorithms, and showed that an asymptotic competitive ratio of 1.63597 is achievable. Ramanan et al. [21] further improved the upper bound to 1.61217. The best known upper bound is that of the Super Harmonic algorithm of Seiden [22] which is 1.58889. As for the lower bound of the competitive ratio, Yao [24] showed that no online algorithm can have an asymptotic competitive ratio less than 1.5. The best known lower bound to date is 1.54014 [23].

For two-dimensional online bin packing, the best known lower bound is 1.907 [3] while the best known upper bound is 2.5545 [13].

For bounded space online bin packing, Csirik and Johnson [6] presented an 1.7-competitive algorithm $\left(K\right.$-Bounded Best Fit algorithms $\left.\left(B B F_{K}\right)\right)$ for one dimensional bin packing using $K$ active bins, where $K \geq 2$. Epstein et al. [8] gave a $1.69103^{d}$-competitive algorithm using $(2 M-1)^{d}$ active bins, where $M \geq 10$ is an integer such that $M \geq 1 /\left(1-(1-\varepsilon)^{1 /(d+2)}\right)-1, \varepsilon>0$ and $d$ is the dimension of the bin packing problem. For the 1-space bounded variant, Fujita [12] gave an $O\left((\log \log m)^{2}\right)$-competitive algorithm, where $m$ is the width of the square bin and the size of each item is $a \times b$, where $a, b$ are integers no larger than $m$. He also proved that the competitive ratio for the 1-bounded space variant is at least 23/11. Recently, Chin et al. [7] proposed an 8.84-competitive packing strategy. Moreover, they proved that the lower bound of the competitive ratio is at least 2.5 .

For the special case where the items are squares, there are also many results $[9-11,14,15,18-20]$. For bounded space online square packing, Epstein and 
van Stee [10] gave a 2.3692-competitive algorithm, they also proved that the lower bound of the competitive ratio is at least 2.36343. Januszewski and Lassak [15] proved that any sequence of square items with a total area of at most 5/16 can be packed into a unit bin. Han et al. [14] studied a variant in which any packed item can be removed so as to guarantee a good competitive ratio and presented a packing algorithm that is 3-competitive. Note that in the above two studies, there is only one bin to pack the square items.

The remaining part of this paper is organized as follows. In Section 2, we propose a 5.155-competitive algorithm for the 1-space bounded bin packing problem; we also prove that the lower bound of the competitive ratio is at least 3. In Section 3, we consider 1-space bounded square packing and give a 4.5-competitive algorithm, which is the first result in this variant. Moreover, we prove that the lower bound of this variant is at least $8 / 3$. In Section 4 , we summarize our results and give some future research directions.

\section{1-Space Bounded 2-Dimensional Bin Packing}

In this section, we propose a packing strategy for 1-space bounded 2-dimensional bin packing with competitive ratio 5.155 . In the previous 8.84-competitive algorithm, the items are classified into three types according to their sizes, and the unit bin is partitioned into two parts: the upper and lower part. The upper part only accommodates items from the two types of the larger sizes, while the lower part only accommodates items from the type of the smallest size. In our new approach, there is no partition in the unit bin which means that an item can be placed at any available position within the bin. This new approach is more flexible than the previous one and can thus achieve better performance.

Since $90^{\circ}$-rotation is allowed, we shall assume that for each rectangular item, the width is no less than the height. We classify the rectangular items into three classes $A, B$ and $C$ according to the width $x$ as follows:

$$
\begin{aligned}
& A=\{(x, y) \mid x \geq 1 / 2\}, \\
& B=\{(x, y) \mid 1 / 4 \leq x<1 / 2\}, \text { and } \\
& C=\{(x, y) \mid x<1 / 4\} .
\end{aligned}
$$

For simplicity, let $A$-item denote an item belonging to class $A$. $B$-item and $C$ item are defined similarly.

In our packing strategy, $A$-items are packed into the active bin using a topdown approach while $B$-items and $C$-items are packed into the bin using a bottom-up approach. If an item cannot be packed into the bin using the strategy, we close the active bin and open a new one to pack the item.

We further divide the $C$-items into subclasses $C_{0}, C_{1}, C_{2}, \ldots$ An item $(x, y)$ belongs to subclass $C_{i}, i \geq 0$, if $2^{-i-1} / 4 \leq x<2^{-i} / 4$. Let $w_{i}$ denote the maximal possible width of items from subclass $C_{i}$. Then, $w_{0}=1 / 4, w_{1}=1 / 8, \ldots$ Each item belonging to subclass $C_{2 j-1}$ or $C_{2 j}(j>0)$ is packed into a row with height $w_{2 j-1}$ and width $1 / 2$. The items from subclasses $C_{2 j-1}$ are packed from left to right while the items from subclass $C_{2 j}$ are packed from right to left in two 
subrows (upper and lower) keeping the lengths of the two subrows balanced at all times (that means a new item is always packed into the shorter subrow). Note that the $C_{j}$-items $(j>0)$ are packed with a $90^{\circ}$-rotation. Figure 2 depicts a row with packed items from subclass $C_{2 j-1}$ and $C_{2 j}$. When handling an item from subclass $C_{2 j-1}$ (or $C_{2 j}$ ), a new row of height $w_{2 j-1}$ must be created if the existing rows of height $w_{2 j-1}$ cannot accommodate this item.

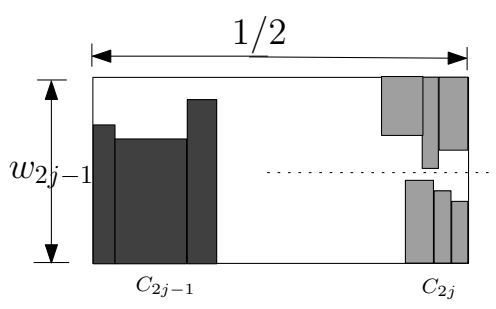

Fig. 2. Packing $C_{2 j-1}$ (or $C_{2 j}$ )-items $(j>0)$ into a row.

Fact 1 For any non-last row of height $w_{2 j-1}$, the occupation ratio is at least $5 / 16$.

Proof. Consider the packing configuration shown in Figure 2. Assuming that the length of the left occupied area (the total height of the packed items from subclass $C_{2 j-1}$ in this row) is $y$, the lengths of the upper and lower subrows in the right occupied area are $y_{1}$ and $y_{2}$ respectively, w.l.o.g., $y_{1} \geq y_{2}$.

If this configuration cannot accommodate the next item from subclass $C_{2 j-1}$ or $C_{2 j}$, we have $y+y_{1}+$ (height of the item) $>1 / 2 \Rightarrow y+y_{1}+1 / 8>1 / 2$. Since the lengths of the two subrows in the right occupied area are balanced, we have $y_{1}-y_{2} \leq w_{2 j} \leq 1 / 16$. Therefore, the total occupied area in this row is at least $\left(y+y_{2}\right) \times w_{2 j-1} / 2$. Since the total area of this row is $w_{2 j-1} / 2$, the occupation ratio is thus at least $y+y_{2} \geq 5 / 16$.

The following is a detailed description of the above packing strategy.

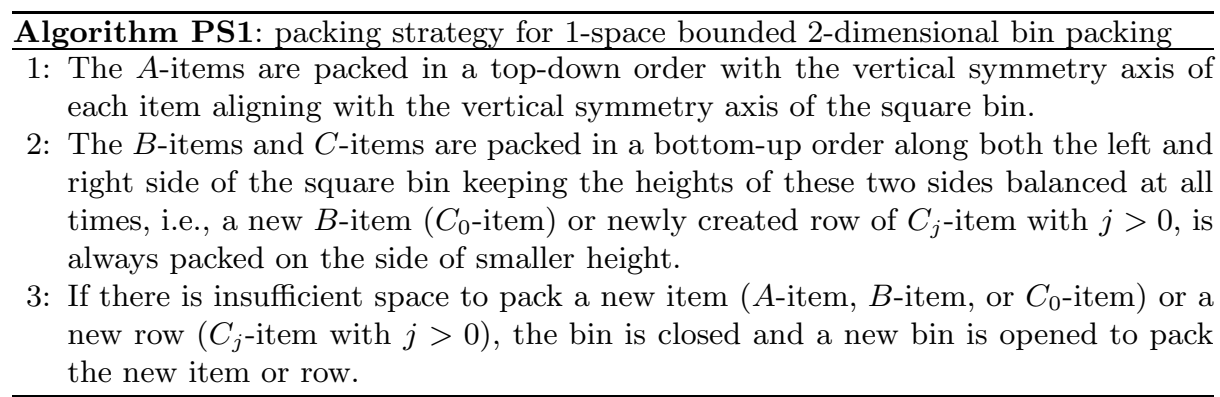

For example, given the current configuration in Figure 3, the height of the packed $A$-items is $y$, the left and right sides of the packed $B$-items and $C$-items are of height $y_{1}$ and $y_{2}$ respectively. 


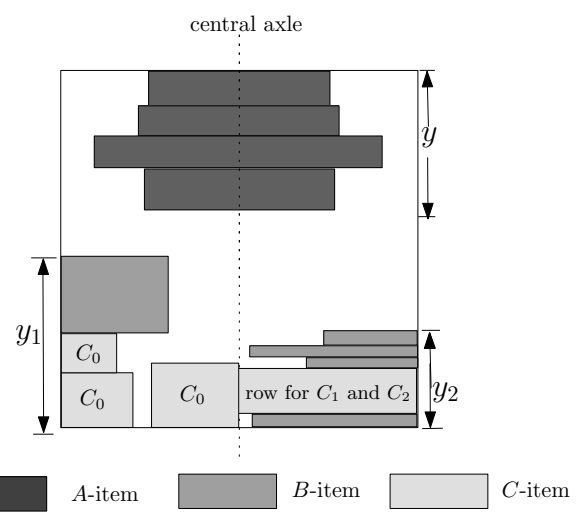

Fig. 3. Packing items into a square bin.

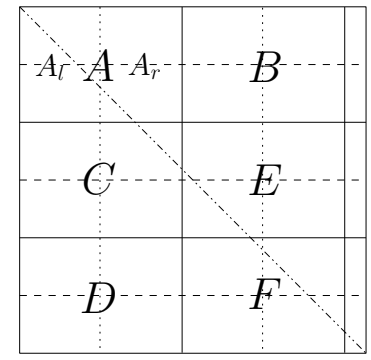

Fig. 4. Partition of unit bin.

Consider packing the $C_{j}$-items with $j>0$. If there are more than one rows for the subclass $C_{j}$, the last row could be almost empty while the occupation ratio of the other rows is at least $5 / 16$ by Fact 1 . Since the last row of each subclass could be almost empty, the total area of such rows is at most $\left(w_{1}+w_{3}+w_{5}+\ldots\right) \times 1 / 2=$ $(1 / 8+1 / 32+\ldots) \times 1 / 2 \approx 1 / 12$.

Theorem 1 The competitive ratio of the packing strategy PS1 is at most 5.155.

Proof. For a given sequence of items, suppose the number of bins used by the packing strategy PS1 is $n$. Let $o_{A}^{i}, o_{B}^{i}$ and $o_{C}^{i}$ be the occupied space of $A$-, $B$ and $C$-items in the $i$-th bin respectively. The average occupation for all the bins is $\sum_{i=1}^{n}\left(o_{A}^{i}+o_{B}^{i}+o_{C}^{i}\right) / n$.

Consider the packing configuration of the $i$-th bin as shown in Figure 3. We have $o_{A}^{i} \geq y / 2, o_{B}^{i} \geq\left(y_{1}+y_{2}-\sum_{j \geq 1} w_{2 j-1}-m\right) / 4 \geq\left(y_{1}+y_{2}-1 / 6-m\right) / 4$, $o_{C}^{i} \geq m \cdot \min \left\{1 / 8, \min _{j>0}\left\{1 / 2-w_{2 j-1}\right\}\right\} \geq m / 8$, where $m$ is the total height of $C_{0}$-items and non-last rows of $C_{j}$-items with $j>0$. Let $p_{C}^{i}=m / 8, q_{C}^{i}=o_{C}^{i}-p_{C}^{i}$.

When $n$ is very large, we have

$$
\frac{\sum_{i=1}^{n}\left(o_{A}^{i}+o_{B}^{i}+o_{C}^{i}\right)}{n} \geq \min _{1 \leq i<n}\left\{o_{A}^{i} / 2+o_{B}^{i} / 2+p_{C}^{i}+o_{A}^{i+1} / 2+o_{B}^{i+1} / 2+q_{C}^{i+1}\right\}
$$

W.l.o.g., assume $y_{1} \geq y_{2}$.

- If the next $A$-item with height $u$ cannot be packed into this bin, we have $y+y_{1}+u>1$ and $o_{A}^{i+1} \geq u / 2$. Thus,

- If $y_{1}-y_{2} \leq 1 / 4$

$$
\begin{aligned}
& o_{A}^{i} / 2+o_{B}^{i} / 2+p_{C}^{i}+o_{A}^{i+1} / 2+o_{B}^{i+1} / 2+q_{C}^{i+1} \\
\geq & y / 4+\left(y_{1}+y_{2}-1 / 6-m\right) / 8+u / 4+m / 8 \\
\geq & y / 4+\left(y_{1}+y_{2}\right) / 8+u / 4-1 / 48 \\
> & \left(1-y_{1}\right) / 4+\left(y_{1}+y_{2}\right) / 8-1 / 48 \\
= & 11 / 48-\left(y_{1}-y_{2}\right) / 8 \\
\geq & 19 / 96
\end{aligned}
$$


- If $y_{1}-y_{2}>1 / 4$, that means the top item in the bottom-left occupied area is a $B$-item. Thus, $o_{B}^{i} \geq\left(y_{2}+y_{2}-1 / 6-m\right) / 4+\left(y_{1}-y_{2}\right)^{2}$. It follows that

$$
\begin{aligned}
& o_{A}^{i} / 2+o_{B}^{i} / 2+p_{C}^{i}+o_{A}^{i+1} / 2+o_{B}^{i+1} / 2+q_{C}^{i+1} \\
\geq & y / 4+\left(y_{2}+y_{2}-1 / 6-m\right) / 8+\left(y_{1}-y_{2}\right)^{2} / 2+u / 4+m / 8 \\
\geq & (y+u) / 4+y_{2} / 4-1 / 48+\left(y_{1}-y_{2}\right)^{2} / 2 \\
> & \left(1-y_{1}\right) / 4+y_{2} / 4-1 / 48+\left(y_{1}-y_{2}\right)^{2} / 2 \\
= & 11 / 48+\left(y_{1}-y_{2}\right)^{2} / 2-\left(y_{1}-y_{2}\right) / 4 \\
\geq & 19 / 96
\end{aligned}
$$

- If the next $B$-item with height $u$ cannot be packed into this bin, we have $y+y_{2}+u>1$ and $o_{B}^{i+1} \geq u^{2}$. Thus

$$
\begin{aligned}
& o_{A}^{i} / 2+o_{B}^{i} / 2+p_{C}^{i}+o_{A}^{i+1} / 2+o_{B}^{i+1} / 2+q_{C}^{i+1} \\
\geq & y / 4+\left(y_{1}+y_{2}-1 / 6-m\right) / 8+u^{2} / 2+m / 8 \\
= & y / 4+\left(y_{1}+y_{2}\right) / 8+u^{2} / 2-1 / 48 \\
\geq & y / 4+y_{2} / 4+u^{2} / 2-1 / 48 \\
> & (1-u) / 4+u^{2} / 2-1 / 48 \\
\geq & 19 / 96
\end{aligned}
$$

- If the next $C$-item with height $u$ cannot be packed into this bin,

- if this item belongs to subclass $C_{i}(i>0)$, then as the width of $w_{i}(i>0)$ is at most $1 / 8$, we must have $y+y_{2}+1 / 8>1$. Thus,

$$
\begin{aligned}
& o_{A}^{i} / 2+o_{B}^{i} / 2+p_{C}^{i}+o_{A}^{i+1} / 2+o_{B}^{i+1} / 2+q_{C}^{i+1} \\
\geq & y / 4+\left(y_{1}+y_{2}-1 / 6-m\right) / 8+m / 8 \\
= & y / 4+\left(y_{1}+y_{2}\right) / 8-1 / 48 \\
\geq & y / 4+y_{2} / 4-1 / 48 \\
> & (1-1 / 8) / 4-1 / 48 \\
= & 19 / 96
\end{aligned}
$$

- if the next item with height $u$ belongs to subclass $C_{0}$, we have $y+$ $y_{2}+u>1$. Note that as this $C_{0}$-item will be packed into the next bin, $q_{C}^{i+1} \geq u^{2}-u / 8$. We thus have,

$$
\begin{aligned}
& o_{A}^{i} / 2+o_{B}^{i} / 2+p_{C}^{i}+o_{A}^{i+1} / 2+o_{B}^{i+1} / 2+q_{C}^{i+1} \\
\geq & y / 4+\left(y_{1}+y_{2}-1 / 6-m\right) / 8+m / 8+\left(u^{2}-u / 8\right) \\
= & y / 4+\left(y_{1}+y_{2}\right) / 8-1 / 48+\left(u^{2}-u / 8\right) \\
\geq & y / 4+y_{2} / 4-1 / 48+\left(u^{2}-u / 8\right) \\
> & (1-u) / 4-1 / 48+\left(u^{2}-u / 8\right) \\
\geq & 149 / 768
\end{aligned}
$$

Combining all of the above cases, we conclude that the competitive ratio of this packing strategy is at most 768/149 $<5.155$.

Theorem 2 The lower bound of competitive ratio for 1-space bounded 2-dimensional bin packing is at least 3. 
Proof. Consider a sequence of items: $S=\left\{X_{1}, X_{2}, \ldots, X_{2 n}, Y_{1}, Y_{2}, Z_{1}, Y_{3}, Y_{4}, Z_{2}, \ldots\right.$, $\left.Y_{n-1}, Y_{n}, Z_{n / 2}, Y_{1}, Y_{2}, Z_{1}, Y_{3}, Y_{4}, Z_{2}, \ldots, Y_{n}\right\}$, in which $\epsilon=o\left(1 / n^{2}\right)$, and

$$
\begin{array}{ll}
X_{2 i-1} & =(1 / 2+i \cdot \epsilon, 1 / 2+i \cdot \epsilon) \\
X_{2 i} & =(1 / 2-(i-1) \cdot \epsilon, 1 / 2-(i-1) \cdot \epsilon) \\
Y_{i} & =(1 / 2+i \cdot \epsilon, 1 / 2-i \cdot \epsilon) \\
Z_{i} & =(1,(2 i+2) \cdot \epsilon)
\end{array}
$$

In the first part of the item sequence containing all the $X_{i}$ items, no online algorithm can pack any two consecutive items into one unit square bin because the sum of the edge lengths of any two consecutive $X$-items is larger than 1 . Thus, at least $2 n$ bins are used for packing all the $X_{i}$ items.

For the remaining part of the item sequence containing all the $Y_{i}$ and $Z_{i}$ items, no three consecutive $Y_{i}$ items with an intervening $Z_{i}$ item can be packed into the same bin. As a result, at least $n$ bins are needed to pack this part of the item sequence.

For optimal offline packing, since $X_{2 i-1}, X_{2 i+2}, Y_{i}, Y_{i}(1 \leq i \leq n-1)$ can be packed into one bin; $X_{2}, Y_{n}, Y_{n}$ can be packed into one bin; $X_{2 n-1}$ can be packed into one bin, and all the $Z_{i}$ items can be packed into one bin, the minimum number of bins needed for packing all the items is at most $n+2$.

From the above analysis, we conclude that no online algorithm can achieve a competitive ratio less than 3 for 1-space bounded 2-dimensional bin packing.

\section{1-Space Bounded Square Packing}

In 1-space bounded square packing, a sequence of square items is to be packed into bins, where there is only one active bin at any time. If a newly arrived square item cannot be packed into the active bin, we close the active bin and open a new one for packing that item and subsequent items. The packing strategy in [14] can be used for this variant directly, leading to a 6-competitive algorithm for 1-space bounded square packing.

Most of the previous studies on square packing use the method of packing square in brick where a brick is a rectangle with aspect ratio $\sqrt{2}$. A brick can be partitioned into two smaller congruent bricks of the same size. Thus, packing a square into a brick can be done recursively. Given a square $Q$, we use $S(Q)$ to denote the smallest brick which can contain $Q$. Let $|R|$ denote the area of rectangle $R$.

We briefly describe the algorithm in [15] for packing a square $Q$ in a brick $B$.

- If there is no empty brick in $B$ of size greater than or equal to $S(Q)$, then give up packing $Q$ in $B$.

- Else pack $Q$ into $B$ as follows:

- if there is an empty brick congruent to $S(Q)$, then pack $Q$ into it,

- else partition the smallest empty brick $P$ that is larger than $S(Q)$ into a sequence of bricks of area $|P| / 2,|P| / 4, \ldots, 2|S(Q)|,|S(Q)|,|S(Q)|$, respectively. Then pack $Q$ into one of the two bricks of area $|S(Q)|$. 
The following lemma is proved in [15].

Lemma 2 If the above algorithm cannot pack an item $Q$ in a brick $B$, then all empty bricks in $B$ are smaller than $S(Q)$. Furthermore, there is at most one empty brick with area $|S(Q)| / 2^{i}$ for each $i=1,2, \ldots$, and the total area of the empty bricks is less than $|S(Q)|$.

Fact 3 If $Q$ is packed in a brick congruent to $S(Q)$, then at least $1 /(2 \sqrt{2})$ of this brick is occupied.

\subsection{A 4.5-Competitive Algorithm}

We partition each unit bin as shown in Figure 4. Note that bricks $A$ to $F$ are of the same size $(1 / 3, \sqrt{2} / 3)$. Each brick can be further partitioned into two congruent bricks. For instance, brick $A$ can be partitioned into $A_{l}$ and $A_{r}$. Our packing strategy is as follows.

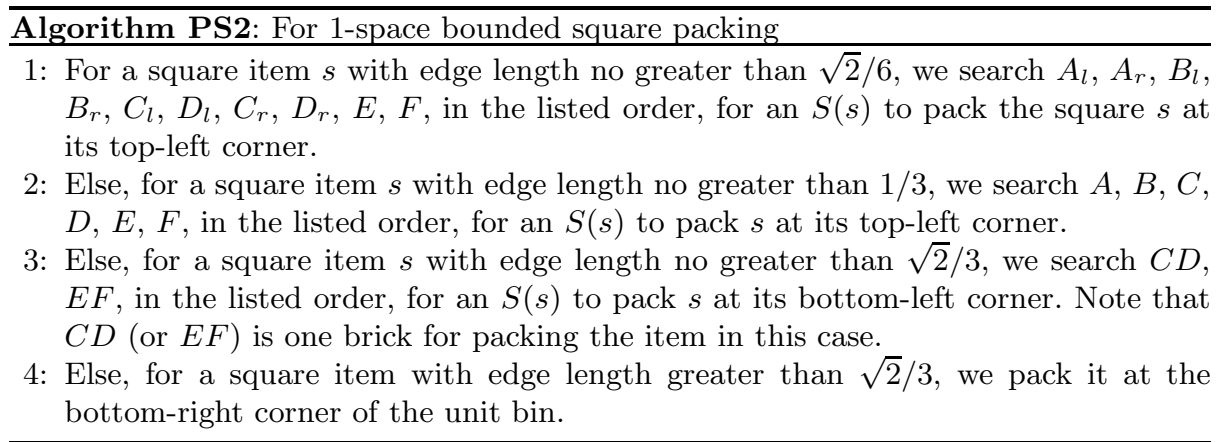

Fact 4 In executing Algorithm PS2, if an item $s$ with edge length no greater than $\sqrt{2} / 6$ is packed in $C_{r}$ or $D_{r}$, the total area of the packed items is larger than $1 / 6$; if an item $s^{\prime}$ with edge length no greater than $\sqrt{2} / 3$ is packed in $E$ or $F$, the total area of the packed items is larger than $2 / 9$.

Proof. According to Algorithm PS2, items with edge length no greater than $\sqrt{2} / 6$ are packed into the bricks $A_{l}, A_{r}, B_{l}, B_{r}, C_{l}, D_{l}, C_{r}, D_{r}$ in the listed order. If $s$ is packed into $C_{r}$ or $D_{r}$, then using Lemma 2, it is easily verified that there is at most one empty brick of area $|S(s)| / 2^{i}(i>0)$ in the preceding bricks. Therefore, by Fact 3, the total occupied area in this bin is at least $(|A|+|B|+$ $\left.\left|C_{l}\right|+\left|D_{l}\right|-\sum_{i>0}|S(s)| / 2^{i}\right) / 2 \sqrt{2}+|s|>1 / 6$. Similarly, if an item $s^{\prime}$ with edge length no greater than $\sqrt{2} / 3$ is packed into $E$ or $F$, the total occupied area is no less than $2 / 9$.

Theorem 3 The competitive ratio of the packing strategy PS2 is at most 4.5.

Proof. To prove this theorem, we will show, based on PS2, that either the total occupied area in a bin is at least $2 / 9$, or the total occupied area in two consecutive bins is at least $4 / 9$. Therefore, the average occupied area in each bin is at least $2 / 9$. Consider the item sequence $s_{1}, s_{2}, \ldots, s_{k-1}, s_{k}, s_{k+1}, \ldots$, where $s_{1}$ is the first item packed into bin $\mathcal{I}$. Suppose $s_{1}$ to $s_{k-1}$ are all packed into $\mathcal{I}$ by PS2, and $s_{k+1}$ is not packed into $\mathcal{I}$. We analyze the situation in packing $s_{k}$. Let $e_{k}$ denote the edge length of $s_{k}$. 
$-e_{k} \geq 2 / 3$

The area of $s_{k}$ is at least $4 / 9$, no matter where $s_{k}$ is packed, the average occupied area in $\mathcal{I}$ and the next bin is thus at least $2 / 9$.

$-1-\sqrt{2} / 3 \leq e_{k}<2 / 3$

In this case, if $s_{k}$ can be packed into $\mathcal{I}$, the occupied area is larger than $2 / 9$. Otherwise, there must be an item in $\mathcal{I}$ preventing $s_{k}$ from being packed into the bottom-right corner.

- If the item is of edge length larger than $\sqrt{2} / 3$ (it must occupy the bottomright corner and cover part of brick $E F$ ), then the occupied area is at least $(\sqrt{2} / 3)^{2}=2 / 9$.

- If the item is of edge length no larger than $\sqrt{2} / 3$ and resides in $E$ or $F$, then according to Fact 4 , the occupied area in $\mathcal{I}$ is at least 2/9.

- If the item is of edge length larger than $\sqrt{2} / 6$ and resides in $C$ or $D$, then from the packing strategy, this item is packed at the top-left corner in $C$ or $D$ whereas $s_{k}$ is packed at the bottom-right corner of $\mathcal{I}$. Therefore, the total edge length of these two items is more than 1 , and the total area of these two items is no less than $(\sqrt{2} / 6+x)^{2}+(1-\sqrt{2} / 6-x)^{2} \geq 1 / 2$, where $\sqrt{2} / 6+x$ is the edge length of the item. Consequently, the average occupied area is at least $1 / 4$, which is larger than $2 / 9$.

- Otherwise, the item is of edge length no greater than $\sqrt{2} / 6$ and resides in $C_{r}$ or $D_{r}$. From Fact 4 , the total area of the packed items is at least $1 / 6$. The total area of items $s_{1}$ to $s_{k}$ is thus at least $1 / 6+(1-\sqrt{2} / 3)^{2}>0.446$, and the average occupied area is larger than $2 / 9$.

$-\sqrt{2} / 3<e_{k}<1-\sqrt{2} / 3$

If $s_{k}$ can be packed into $\mathcal{I}$, the occupied area in this bin is at least $(\sqrt{2} / 3)^{2}=$ $2 / 9$. Otherwise, packing $s_{k}$ at the bottom-right corner of the bin results in $s_{k}$ overlapping some packed item in brick $E$ or $F$. If one of the packed items is of edge length no less than $\sqrt{2} / 3$, then the total occupied area in the bin is at least $2 / 9$. Otherwise, from Fact 4 , the total occupied area contributed by the items $s_{1}, s_{2}, \ldots, s_{k-1}$ is at least $2 / 9$.

$-e_{k} \leq \sqrt{2} / 3$

If $s_{k}$ cannot be packed into this bin, then similar to the above case, the occupied area in this bin is at least $2 / 9$.

Otherwise, $s_{k}$ can be packed into $\mathcal{I}$. But $s_{k+1}$ cannot be packed into this bin. Again, similar to the above analysis, the average occupied area in $\mathcal{I}$ is at least $2 / 9$.

Since the average occupied area in each bin is at least $2 / 9$, the competitive ratio of our packing strategy PS2 is thus at most 4.5.

\subsection{Lower Bound of The Competitive Ratio}

Now we shall derive a lower bound of the competitive ratio for 1-space bounded square packing. Roughly speaking, we use three types of items to derive the lower bound. 
- Type- $X$ has area slightly larger than $1 / 4$.

- Type- $Y$ has area slightly smaller than $1 / 4$.

- Type- $Z$ has sufficiently small area.

The high level idea underlying the lower bound proof is as follows: We construct a sequence with $n$ items of type- $X, 3 n$ items of type- $Y$ and $2 n / 3$ items of type- $Z$ such that we need $n$ bins to pack the first $n$ items of type- $Y, n$ bins to pack the $n$ items of type- $X$, and $2 n / 3$ bins to pack the remaining $2 n$ items of type- $Y$ and $2 n / 3$ items of type- $Z$. But for the optimal packing strategy, $n+2$ bins is sufficient. Specifically, one bin for packing the $2 n / 3$ items of type- $Z, n+1$ bins for packing all the type- $X$ and type- $Y$ items; in most of these bins three type- $Y$ items and one type- $X$ item are packed together in one bin.

Theorem 4 There is no online algorithm with a competitive ratio less than $8 / 3$ for 1-space bounded square packing.

Proof. Consider a sequence of items $Y_{1}, X_{1}, Y_{2}, X_{2}, \ldots, Y_{n}, X_{n}, Y_{n}, Y^{\prime}, Y^{\prime}, Z$, $3 Y^{\prime}, Z, 3 Y^{\prime}, Z \ldots$, containing $n$ type- $X$ items, $3 n$ type- $Y$ items and $2 n / 3$ type- $Z$ items. Let $\epsilon=o\left(1 / n^{2}\right)$.

$$
\begin{aligned}
& X_{i}=(1 / 2+(n+2-i) \cdot \epsilon, 1 / 2+(n+2-i) \cdot \epsilon) \\
& Y_{i}=(1 / 2-(n+1-i) \cdot \epsilon, 1 / 2-(n+1-i) \cdot \epsilon) \\
& Y^{\prime}=(1 / 2-n \cdot \epsilon, 1 / 2-n \cdot \epsilon) \\
& Z=(3 n \cdot \epsilon, 3 n \cdot \epsilon)
\end{aligned}
$$

For the first part of the item sequence containing $2 n$ items, any two consecutive items cannot be packed together into one bin. Thus, any online algorithm will use at least $2 n$ bins for the first part.

For the remaining part containing $2 n$ type- $Y$ items and $2 n / 3$ type- $Z$ items, any four consecutive type- $Y$ items cannot be packed together into one bin because of the intervening type- $Z$ item. Thus, any online algorithm will use at least $2 n / 3$ bins to pack the remaining items.

The total number of bins used by any online algorithm is at least $8 n / 3$.

For offline optimal packing, $Y_{i}, X_{i+1}, Y^{\prime}, Y^{\prime}(i<n)$ can be packed together into one bin. $X_{1}$ can be packed into the $n$-th bin, while $Y_{n}, Y_{n}, Y^{\prime}$ can be packed into the $(n+1)$-th bin. The type- $Z$ items can all be packed into the $(n+2)$-th bin. Thus, the minimum number of bins used for packing all the items is at most $n+2$.

Hence, the lower bound of competitive ratio is at least $8 / 3$.

\section{Concluding Remarks}

We have studied 1-space bounded 2-dimensional bin packing and presented online algorithms for rectangle packing and square packing. For rectangle packing, we derived an upper bound of 5.155 and a lower bound of 3. For square packing, the corresponding bounds we derived are 4.5 and 2.667. These bounds surpass the previously best known bounds. We feel that the gap between the upper and lower bound is quite big in both cases. We thus propose closing these gaps as open problems for future research. 


\section{References}

1. N. Bansal, J.R. Correa, C. Kenyon and M. Sviridenko. Bin Packing in Multiple Dimensions: In-approximability Results and Approximation Schemes. Mathematics of Operations Research, 31(1): 31-49, 2006.

2. N. Bansal, A. Caprara and M. Sviridenko. Improved approximation algorithm for multidimensional bin packing problems, FOCS 2006: 697-708.

3. D. Blitz, A. van Vliet, and G. J. Woeginger. Lower bounds on the asymptotic worst-case ratio of on-line bin packing algorithms. unpublished manuscript, 1996.

4. A. Caprara. Packing 2-dimensional bins in harmony. FOCS 2002: 490-499.

5. F.R.K. Chung, M.R. Garey, D.S. Johnson. On packing two-dimensional bins. SIAM J. Algebraic Discrete Methods, 3(1):66-76, 1982.

6. J. Csirik and D.S. Johnson. Bounded Space On-Line Bin Packing: Best is Better than First. Algorithmica (2001) 31: 115-138.

7. Francis Y.L. Chin, Hing-Fung Ting, and Yong Zhang. 1-Space Bounded Algorithms for 2-Dimensional Bin Packing. In Proc. of the 20th Annual International Symposium on Algorithms and Computation (ISAAC 2009), LNCS 5878, 321-330.

8. Leah Epstein, Rob van Stee. Optimal Online Algorithms for Multidimensional Packing Problems. SIAM Jouranl on Computing, 35(2), 431-448, 2005.

9. Leah Epstein, Rob van Stee. Online square and cube packing. Acta Inf. 41(9): 595-606 (2005)

10. Leah Epstein, Rob van Stee. Bounds for online bounded space hypercube packing. Discrete Optimization 4(2007): 185-197.

11. C.E. Ferreira, E.K. Miyazawa, and Y. Wakabayashi. Packing squares into squares. Pesquisa Operacional, 1999, 19: 223-237.

12. S. Fujita. On-Line Grid-Packing with a Single Active Grid. Information Processing Letters 85(2003) 199-204.

13. Xin Han, Francis Chin, Hing-Fung Ting, Guochuan Zhang, Yong Zhang. A New Upper Bound on 2D Online Bin Packing. manuscript.

14. Xin Han, Kazuo Iwama and Guochuan Zhang Online removable square packing. Theory of Computing Systems, pp. 38-55, vol 43(1), July, 2008

15. J. Januszewski and M. Lassak. On-line packing sequences of cubes in the unit cube. Geometriae Dedicata 1997, 67: 285-293.

16. D.S. Johnson, A.J. Demers, J.D. Ullman, M.R. Garey, R.L. Graham. Worst-Case performance bounds for simple one-dimensional packing algorithms. SIAM Journal on Computing 3(4), 299-325 (1974).

17. C. C. Lee and D. T. Lee. A simple on-line bin packing algorithm. J. Assoc. Comput. Mach., 32:562-572, 1985.

18. J.Y.-T. Leung, T.W. Tam, C.S. Wong, G.H. Young, and F.Y.L. Chin. Packing squares into a square. J. Parallel Distrib. Comput. 1990, 10: 271-275.

19. Y. Kohayakawa, F.K. Miyazawa, P. Raghavan, and Y. Wakabayashi. Multidimensionalcube packing. Algorithmica, 40(3) 173-187,2004.

20. A.Meir and L. Moser. On packing of squares and cubes. Journal of combinatorial theory, 1968, 5: 126-134.

21. P.V.Ramanan, D.J. Brown, C.C. Lee, D.T. Lee, On-line bin packing in linear time. Journal of Algorithms, 10, 305-326 (1989).

22. S.S. Seiden, On the online bin packing problem, J. ACM 49, 640-671, 2002

23. A. van Vliet. An improved lower bound for on-line bin packing algorithms. Information Processing Letters, 43:277-284, 1992.

24. A.C.-C. Yao, New Algorithms for Bin Packing. Journal of the ACM 27, 207-227, (1980). 